\title{
Anesthesia for massive retrosternal goiter with severe intrathoracic tracheal narrowing: the challenges imposed -A case report-
}

\author{
Peter Chee Seong Tan, and Norzalina Esa \\ Department of Anesthesiology and Intensive Care, Sarawak General Hospital, Kuching, Sarawak, Malaysia
}

\begin{abstract}
Anesthetic management of patients with mediastinal masses remains challenging as acute cardiorespiratory decompensation may follow induction of anesthesia. We describe a 57 year old lady with massive retrosternal goiter and severe intrathoracic tracheal compression who had a total thyroidectomy. Comprehensive contingency plans were an essential prerequisite for successful management of difficult airway, including multidisciplinary involvement of otorhinolaryngologic and cardiothoracic surgeons preparing for rigid bronchoscopy and cardiopulmonary bypass. Awake oral fiberoptic intubation was performed under dexmedetomidine sedation. Severe tracheal narrowing necessitated usage of a $5.0 \mathrm{~mm}$ uncuffed flexometallic endotracheal tube. Anesthesia was maintained with sevoflurane and dexmedetomidine infusion with target controlled infusion of remifentanil as analgesia. No muscle relaxant was given. Surgical manipulation led to intermittent total tracheal compression and inadequate ventilation. The tumor was successfully removed via the cervical approach. A close working relationship between anesthesiologists and surgeons was the key to the safe use of anesthesia and uneventful recovery of this patient.
\end{abstract} (Korean J Anesthesiol 2012; 62: 474-478)

Key Words: Awake fiberoptic intubation, Dexmedetomidine, Difficult airway, Mediastinal mass.

Anesthetic management of patients with mediastinal mass remains a formidable challenge. This group of patients is heterogenous with respect to their clinical evolution as well as with their various types, sizes and localization of masses [1]. There are different ways in which vital organs in the limited mediastinal space may be affected. Therefore, the respiratory and hemodynamic responses to anesthesia may vary among individuals. There are sporadic case reports which illustrate acute cardiorespiratory decompensation in the course of anesthesia due to tumor-related compression of mediastinal organs [2-4] which often resulted in life-threatening conditions or sometimes fatal outcomes. We describe the anesthetic management of a massive retrosternal goiter with severe intrathoracic tracheal narrowing scheduled for total

Received: April 18, 2011. Revised: May 8, 2011. Accepted: May 9, 2011.

Corresponding author: Peter Chee Seong Tan, M.D., Department of Anesthesiology and Intensive Care, Sarawak General Hospital, Kuching, Sarawak 93586, Malaysia. Tel: 6082276428, Fax: 6082419495, E-mail: petecstan@yahoo.com

(c) This is an open-access article distributed under the terms of the Creative Commons Attribution Non-Commercial License (http:// creativecommons.org/licenses/by-nc/3.0/), which permits unrestricted non-commercial use, distribution, and reproduction in any medium, provided the original work is properly cited. 
thyroidectomy.

\section{Case Report}

A 57 year old lady $(153 \mathrm{~cm}, 78 \mathrm{~kg})$ complained of progressively enlarged anterior neck swelling for the past eight years. No dysphagia, cough, dyspnea, noisy breathing, hoarseness or syncope was elicited, even when changing from a vertical to supine position. She was taking carbimazole (20 mg twice daily) and propanolol (40 mg twice daily) which were started two years ago when she was found to be clinically and biochemically thyrotoxic during her first presentation [thyroxine (T4) 164.00 $\mathrm{pmol} / \mathrm{L}$, thyroid stimulating hormone $(\mathrm{TSH})<0.005 \mathrm{mIU} / \mathrm{L}]$. No symptoms were suggestive of hyper- or hypothyroidism upon review. She was hypertensive and taking amlodipine (10 mg once daily), hydrochlorothiazide ( $12.5 \mathrm{mg}$ once daily) and prazosin (1 mg three times daily).

Examination revealed normal vital signs. A firm neck swelling measuring $12 \times 25 \mathrm{~cm}$ with dilated superficial veins was found
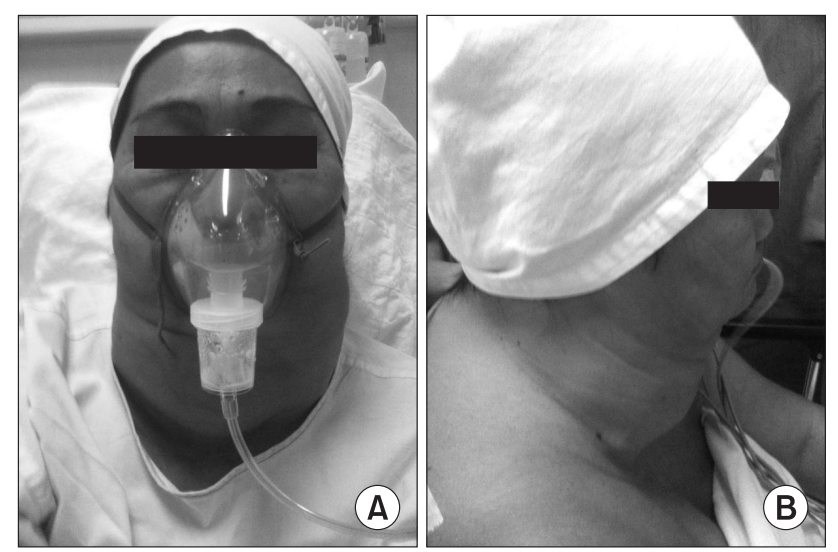

Fig. 1. (A) Anterior view of neck. (B) Lateral view of neck. to extend below the chin retrosternally (Fig. 1). No bruit was heard and no edema of the upper body was elicited. She was euthyroid clinically. Neck flexion and rotation were minimal due to the huge mass, but she had adequate mouth opening and the Mallampati score was 2. Indirect laryngoscopy noted normal vocal cords. Her systemic examination showed no other abnormalities.

Blood count, renal profile and arterial blood gas were normal. The latest thyroid function test revealed T4 of $7.26 \mathrm{pmol} / \mathrm{L}$ and $\mathrm{TSH}$ of $0.37 \mathrm{mIU} / \mathrm{L}$. Chest radiograph noted a large thyroid mass extending retrosternally to the level of the carina on the right side. The trachea was narrowed and displaced to the left side. Computed tomographs (CT) of neck and thorax confirmed the presence of severely enlarged thyroid glands. Both lobes of the thyroid gland extended superiorly to the level of the mandible. Inferiorly, the left lobe of thyroid gland extended to the thoracic inlet and the right lobe of thyroid gland went down into the right side of the mediastinum to the level of the right main bronchus. The surrounding structures with fairly clear tissue planes in between were displaced. The major neck vessels and superior vena cava were patent. There was severe tracheal narrowing $7 \mathrm{~cm}$ from the arytenoids or vocal cords at the level of C7 vertebrae (Fig. 2-4). The anteroposterior diameter at the narrowest point was $12 \mathrm{~mm}$ and its transverse diameter was $6 \mathrm{~mm}$. There was a gradual widening beyond this point with normal airway diameter at the carinal region and the main bronchi. No mass infiltration into the airway was found.

Comprehensive contingency plans were developed preoperatively involving a general surgeon, cardiothoracic surgeon, otorhinolaryngologic (ORL) surgeon and anesthesiologists emphasizing on the management of difficult airway and acute cardiorespiratory decompensation following induction of anesthesia. The ORL surgeon was on standby in the operating room to initiate rigid bronchoscopy and jet ventilation whereas

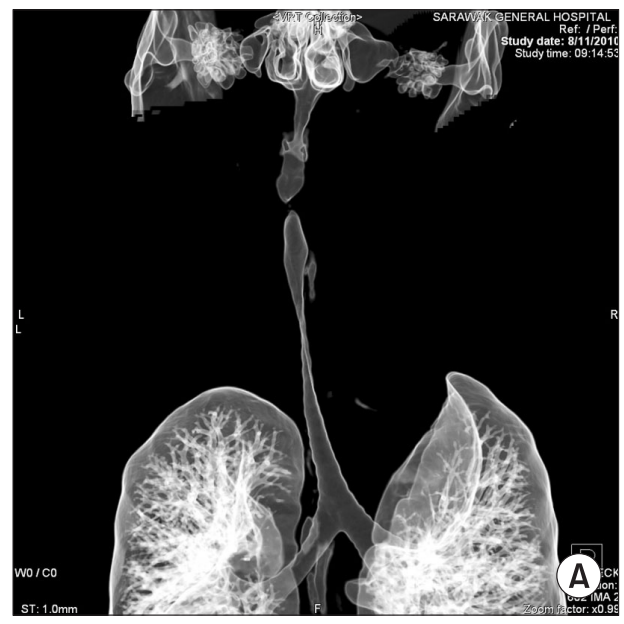

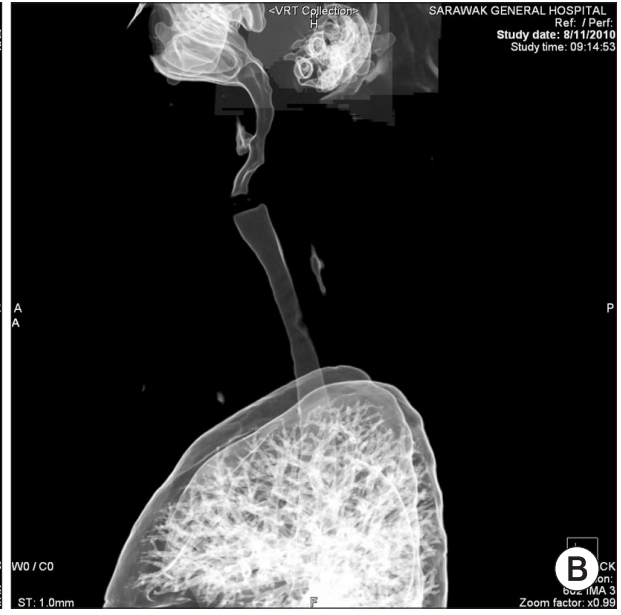

B.
Fig. 2. (A) Coronal view of neck shows transverse diameter of $6 \mathrm{~mm}$ at the narrowest point of tracheal compression. (B) Sagittal view of neck shows anteroposterior diameter of $12 \mathrm{~mm}$ at the narrowest point of tracheal compression at the level of C7 vertebra. 

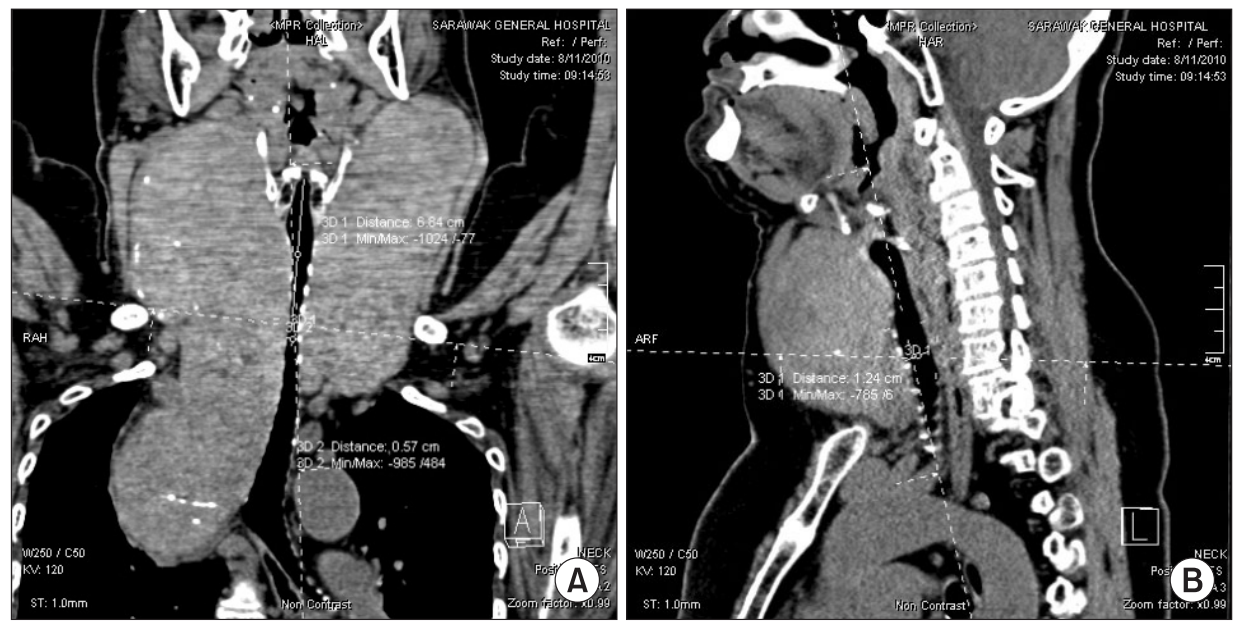

Fig. 3. (A) Coronal view of neck shows transverse diameter of $6 \mathrm{~mm}$ at the narrowest point of tracheal compression. (B) Sagittal view of neck shows anteroposterior diameter of $12 \mathrm{~mm}$ at the narrowest point of tracheal compression at the level of $\mathrm{C} 7$ vertebra.

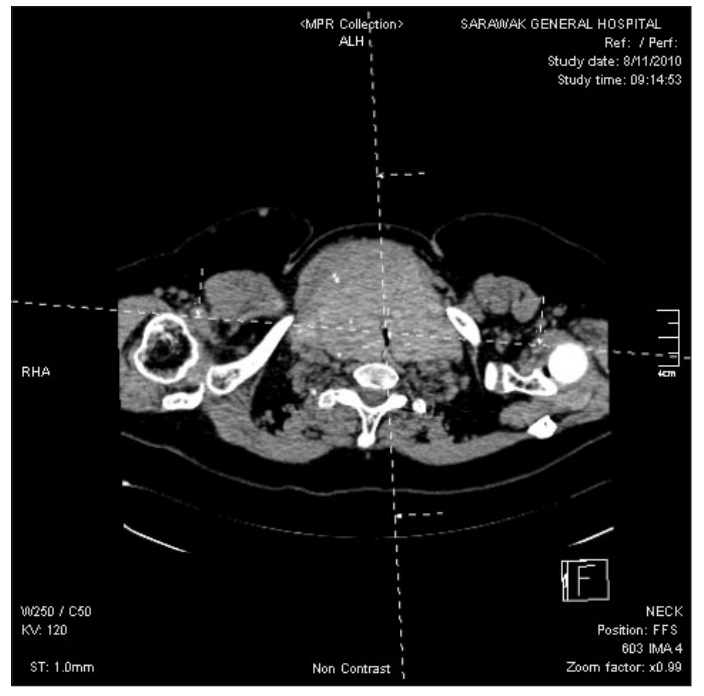

Fig. 4. Cross-sectional view of neck shows the narrowest point of tracheal compression.

the cardiothoracic surgeon and perfusionist prepared for cardiopulmonary bypass (CPB) as rescue in the event of failed airway control or a severe cardiovascular event. A written anesthetic consent was taken with explanation focused on risks of difficult airway, consequences of hypoxia and mediastinal mass syndrome following induction of anesthesia. A preoperative fasting of six hours for solid food and two hours of clear fluid was followed. Premedication consisted of nebulized lignocaine $(2 \%, 60 \mathrm{mg})$ to provide airway anesthesia and intravenous (IV) glycopyrrolate $(200 \mathrm{mcg}$ ) as an antisialagogue to prepare for awake flexible fiberoptic intubation (FOI). No sedative premedication was given.

The left radial artery was cannulated under local anesthesia and the patient was put on American Society of Anesthesiologists standard monitoring with addition of entropy (GE Healthcare,
Finland Oy) to monitor the depth of anesthesia. Awake FOI was performed under infusion of dexmedetomidine titrated at $0.2-$ $0.3 \mathrm{mcg} / \mathrm{kg} / \mathrm{h}$ without the administration of a prior loading dose. Once the glottic structures were identified, $2 \%$ lignocaine (40 mg) was sprayed directly onto the glottic inlet. Another $40 \mathrm{mg}$ of $2 \%$ lignocaine was sprayed below the vocal cords to provide further airway anesthesia. Esmolol (60 mg, IV) was administered to obtund the sympathetic response. Tracheal intubation was done with $5.0 \mathrm{~mm}$ uncuffed flexometallic tube, the largest inner diameter of tracheal tube possible in view of severe tracheal narrowing. However, it resulted in excessive gas leaking necessitating pharyngeal packing and high gas flow up to $8-10 \mathrm{~L} / \mathrm{min}$ to achieve a tidal volume of approximately $350 \mathrm{ml}$. No deterioration in hemodynamics or difficulty in delivering positive pressure ventilation was demonstrated following administration of propofol $(60 \mathrm{mg}$, IV) and sevoflurane at minimum alveolar concentration of 1.0 after intubation. Her blood pressure and heart rates were maintained within $15 \%$ of the baseline values. Cannulation of the left femoral vein with triple lumen catheter 7 French was then performed. Anesthesia was maintained with sevoflurance and dexmedetomidine infusion at $0.2-0.3 \mathrm{mcg} / \mathrm{kg} / \mathrm{h}$ and target controlled infusion (TCI) of remifentanil (Space pump, B. Braun, Germany) with effect site targeting at $2-4 \mathrm{ng} / \mathrm{ml}$ as analgesia. Response entropy (RE) and state entropy (SE) read 35 to 45 and 30 to 40 , respectively. No muscle relaxant was given throughout the procedure. Surgical manipulation led to intermittent total tracheal compression and inadequate ventilation but was not complicated with desaturation. The 1.3 kg tumor was successfully removed via cervical approach with minimal blood loss and the trachea was patent, negating the need for elective tracheostomy. Direct laryngoscopy performed at the end of surgery demonstrated a Cormack and Lehane grade one view of the glottic inlet and allowed for easy changing 
to polyvinyl chloride (PVC) endotracheal tube. The patient was extubated with all due precautions after overnight ventilation under sedation with dexmedetomidine at $0.2 \mathrm{mcg} / \mathrm{kg} / \mathrm{h}$. No respiratory compromise was noted after extubation and she had an uneventful recovery.

\section{Discussion}

Mediastinal mass syndrome (MMS) describes a clinical condition caused by a mediastinal mass in patients undergoing anesthesia [5]. It can occur at every stage of anesthesia up to the postoperative period [6] or even simply by a change of posture [7]. Acute respiratory and/or cardiovascular decompensation can occur. Direct mechanical compression of the trachea, main bronchi or both by the tumor may lead to total airway occlusion whereas external compression of major vessels (i.e. the pulmonary artery and superior vena cava) or even the heart may result in cardiovascular catastrophe which can be potentially fatal.

A review of 98 adult patients with anterior or middle mediastinal masses who had undergone over one hundred anesthetics demonstrated perioperative cardiorespiratory complications $14.3 \%$ of the time [6]. The cases mainly comprised of lymphoma (21.0\%), followed by thymoma (15.3\%). Only $8.2 \%$ of these patients presented with an intrathoracic goiter and thyroid carcinoma. The authors concluded the presence of cardiorespiratory symptoms and signs at presentation are associated with perioperative complications. Other high risk factors are the presence of pericardial effusion or tracheal compression of greater than $50 \%$ as well as mixed restrictive and obstructive patterns on pulmonary function tests. However, none of the patients experienced airway obstruction during anesthesia despite some cases having severe compression of the tracheobronchial tree or peak expiratory flow rates of less than $40 \%$ of predicted values. We did not subject our patient to dynamic tests like flow-volume curves in relation to positional change as the patient was asymptomatic despite CT evidence of critical airway narrowing. In fact, Slinger and Karsli [8] proposed that the assessment is not beneficial as flow-volume loops have been shown to be poorly correlated with the degree of airway obstruction and have not demonstrated usefulness in management. Nevertheless, the challenges of anesthetizing this patient could not be underestimated.

Erdös and Tzanova [5] classified possible anesthetic MMSrisk into three groups according to the clinical symptoms and diagnostic results. The patients are 'safe' if they are asymptomatic whereas the presence of MMS signs and positive diagnostic evaluation are assigned in the 'unsafe' group. The 'uncertain' group is in between the former two categories and comprises of patients with moderate clinical symptoms or asymptomatic but with CT evidence of tracheobronchial tree obstruction (i.e. diameter less than $50 \%$ of normal) or abnormal dynamic evaluation. Nevertheless, the authors suggested adequate staff on hand prior to induction of anesthesia including the presence of a second anesthesiologist and a perfusionist for those patients who are identified as 'unsafe' and 'uncertain. Our patient belonged to the latter group, thus we were aware of the need for close interdisciplinary cooperation in managing this patient, including input from the endocrinologist in optimization of her thyroid status. Comprehensive contingency plans on airway management had been developed with the involvement of anesthesiologists, a general surgeon, an ORL and cardiothoracic surgeons. The availability of rigid bronchoscopy with jet ventilation would offer rescue in the event of loss of airway control [9]. Temporary airway stenting via rigid bronchoscopy has also been described in the literature for patients presenting with mediastinal masses [10], but our institution is not equipped with airway stents. In addition, cardiopulmonary bypass (CPB) would be life-saving during acute cardiorespiratory decompensation [4]. However, femoral cannulation was not achieved under local anesthesia preoperatively which we reserve for the 'unsafe' patients. Though several authors advocate a primed CPB machine and femoral cannulae in-situ prior to inducing the 'unsafe' patients $[5,11,12]$, there are no standard guidelines for management of extracorporeal circulation in the 'uncertain' category. Nevertheless, a prepared CPB machine and a perfusionist were within the vicinity of our operating room.

On the other hand, positioning change may help to relieve the mass effect of tumors [7]. Induction was done on an operating table that enabled immediate changes in the patient's position. It is vital to identify the most 'comfortable' position in terms of respiration and hemodynamics in those symptomatic patients prior to surgery [5]. The tumor-related compression pressure is usually lowest in this position and hence the decompensation may be readily corrected or minimized. A tiltable operating table also allows checking for obstruction of airways in relation to positioning changes via the bronchoscope.

Awake FOI was performed under sedation with dexmedetomidine. Awake FOI and awake tracheostomy are the recommended approaches in managing anticipated difficult airways but it was apparent that the latter was not feasible in this case. The former offers low potential for airway loss but the patient's cooperation is essential. We provided airway anesthesia via nebulized lignocaine but the presence of huge neck mass negated the application of airway local anesthetic blocks. Therefore, discomfort during airway manipulation would be undesirable as it might lead to potential hemodynamic swings in a hypertensive patient. The administration of dexmedetomidine infusion was indeed helpful albeit smaller hemodynamic perturbations might still occur. Dexmedetomidine is an alpha-2 
adrenoceptor agonist with sedative, anxiolytic and analgesic properties, yet it is devoid of respiratory depression. It has been shown to provide a conducive intubating condition during awake FOI [13] which offers better patient tolerance, better preservation of patent airways and more hemodynamic stability. These features have been illustrated in our case too where the changes in hemodynamics were within the narrow range of baseline values and the achieved RE and SE were 60 and 55, respectively during airway manipulation.

No muscle relaxant was given throughout the procedure in view of a possible airway collapse following neuromuscular blockade [5]. Spontaneous respiration preserves the negative intrapleural pressure which maintains the airway diameter. In fact, spontaneous respiration had been allowed successfully in a patient with mediastinal mass undergoing thoracotomy [14]. We maintained anesthesia with sevoflurane guided by entropy. Excessive gas leaking made monitoring depth of anesthesia essential. Requirements of inhalational anesthetic were further reduced with the concurrent administration of dexmedetomidine and TCI remifentanil. Continuation of dexmedetomidine infusion as sedation in the intensive care unit offered an awake patient to be extubated early. Our preferred choice of opiate was remifentanil due to its very short half-life. Overall, the general principles for safe anesthesia of patients with mediastinal masses were applied. Rapid control of general anesthesia to maintain normal muscle tone and adequate spontaneous respiration via the administration of short acting medications were as recommended [5]. Nevertheless, there is always a need to individualize management on a case-by-case basis.

In conclusion, a comprehensive preoperative planning and a close working relationship among multidisciplinary medical teams were prerequisite for successful delivery of anesthesia and uneventful recovery of this patient. A structured in-house clinical protocol should be developed and deployed in order to ensure safe management of such patients in the future.

\section{References}

1. Yoneda KY, Louie S, Shelton DK. Mediastinal tumors. Curr Opin
Pulm Med 2001; 7: 226-33.

2. Keon TP. Death on induction of anesthesia for cervical biopsy. Anesthesiology 1981; 55: 471-2.

3. Lin CM, Hsu JC. Anterior mediastinal tumour identified by intraoperative transesophageal echocardiography. Can J Anaesth 2001; 48: 78-80.

4. Asai T. Emergency cardiopulmonary bypass in a patient with a mediastinal mass. Anaesthesia 2007; 62: 859-60.

5. Erdös G, Tzanova I. Perioperative anaesthetic management of mediastinal mass in adults. Eur J Anaesthesiol 2009; 26: 627-32.

6. Béchard P, Létourneau L, Lacasse Y, Côtè D, Bussières JS. Perioperative cardiorespiratory complications in adults with mediastinal mass: incidence and risk factors. Anesthesiology 2004; 100: 826-34.

7. Choi WJ, Kim YH, Mok JM, Choi SI, Kim HS. Patient repositioning and the amelioration of airway obstruction by an anterior mediastinal tumor during general anesthesia-A case report. Korean J Anesthesiol 2010; 59: 206-9.

8. Slinger P, Karsli C. Management of the patient with a large anterior mediastinal mass: recurring myths. Curr Opin Anaesthesiol 2007; 20: 1-3.

9. Chao YK, Liu YH, Hsieh MJ, Wu YC, Liu HP, Wang CJ, et al. Controlling difficult airway by rigid bronchoscope- an old but effective method. Interact Cardiovasc Thorac Surg 2005; 4: 175-9.

10. Gothard JW. Anesthetic considerations for patients with anterior mediastinal masses. Anesthesiol Clin 2008; 26: 305-14.

11. Tempe DK, Arya R, Dubey S, Khanna S, Tomar AS, Grover V, et al. Mediastinal mass resection: femorofemoral cardiopulmonary bypass before induction of anesthesia in the management of airway obstruction. J Cardiothorac Vasc Anesth 2001; 15: 233-6.

12. Sendasgupta C, Sengupta G, Ghosh K, Munshi A, Goswami A. Femoro-femoral cardiopulmonary bypass for the resection of an anterior mediastinal mass. Indian J Anaesth 2010; 54: 565-8.

13. Tsai CJ, Chu KS, Chen TI, Lu DV, Wang HM, Lu IC. A comparison of the effectiveness of dexmedetomidine versus propofol targetcontrolled infusion for sedation during fibreoptic nasotracheal intubation. Anaesthesia 2010; 65: 254-9.

14. Sibert KS, Biondi JW, Hirsch NP. Spontaneous respiration during thoracotomy in a patient with a mediastinal mass. Anesth Analg 1987; 66: 904-7. 